\title{
GESTÃO DE MUDANÇA EM PROJETOS DE INOVAÇÃO: RSL TERCIÁRIA DE PUBLICAÇOES OPEN ACCESS
}

\author{
Fabíola Amaral \\ Universidade Federal do Rio de Janeiro/COPPE
}

Rua Horácio Macedo, Bloco G - Cidade Universitária, 68507, Rio de Janeiro/RJ 21941-972

fabiola.amaral@ufrj.br

\section{Raquel Faraco}

Universidade Federal do Rio de Janeiro/COPPE

Rua Horácio Macedo, Bloco G - Cidade Universitária, 68507, Rio de Janeiro/RJ 21941-972 rmfaraco@pep.ufrj.br

\section{David Campos}

Universidade Federal do Rio de Janeiro/COPPE

Rua Horácio Macedo, Bloco G - Cidade Universitária, 68507, Rio de Janeiro/RJ 21941-972 davidcampos13@gmail.com

\section{Denise Ferreira}

Universidade Federal do Rio de Janeiro/COPPE

Rua Horácio Macedo, Bloco G - Cidade Universitária, 68507, Rio de Janeiro/RJ 21941-972 denise.sf70@gmail.com

\section{Tharcisio Cotta Fontainha}

Universidade Federal do Rio de Janeiro/COPPE

Rua Horácio Macedo, Bloco G - Cidade Universitária, 68507, Rio de Janeiro/RJ 21941-972

fontainha@pep.ufrj.br

\section{RESUMO}

A gestão da mudança pode ser uma ferramenta importante para superar as dificuldades na implementação de projetos de inovação, com a intenção de minimizar a resistência dos funcionários a todas as medidas que serão impostas para de fato conseguir minimizar o impacto e solucionar os possíveis problemas. Através de uma revisão sistemática da literatura, o objetivo do artigo é identificar as recomendações mais recentes, comuns aos diferentes campos de estudo sobre a gestão da mudança em projetos de inovação, mesmo em áreas multidisciplinares. A pesquisa analisou 6 artigos dos 51 préselecionados e encontrou recomendações gerais e lacunas para futuras pesquisas sobre o processo de inovação.

Palavra-chave: gestão de mudança; inovação; revisão de literatura; estratégia; organizacional. 


\begin{abstract}
Change management can be an important tool to overcome difficulties of innovation projects, with the intention of minimizing the resistance of employees to all the measures that will be imposed in order to actually minimize the impact and solve the possible problems. Through a systematic literature review, the aim of the research is to identify the most recent recommendations, common to the different fields of study on change management in innovation projects, even in multidisciplinary areas. The research analyzed 6 articles out of 51 pre-selected and found many recommendations and gaps for future research on the innovation process.
\end{abstract}

Keywords: change management; innovation; literature review; strategy; organizational.

\title{
Como Citar:
}

AMARAL, Fabíola; FARACO, Raquel; CAMPOS David; FERREIRA Denise; FONTAINHA Tharcisio. Gestão da Mudança em Projetos de Inovação: RSL Terciária de Publicações Open Access In: SIMPÓSIO DE PESQUISA OPERACIONAL E LOGÍSTICA DA MARINHA, 19. 2019, Rio de Janeiro, RJ. Anais [...]. Rio de Janeiro: Centro de Análises de Sistemas Navais, 2019.

\section{INTRODUÇÃO}

Mudança e morte, as únicas certezas de qualquer pessoa, em qualquer lugar, em qualquer época. Mudamos de corpo e comportamento durante toda a nossa vida, mudamos o modo de pensar, às vezes drasticamente. Mudamos de casa, de bairro, de estado, de país... atualmente já se estuda a mudança de planeta, num futuro nem tão distante. Segundo CURVELLO (2002), uma mudança (seja estrutural, gerencial ou cultural) só se realiza quando os indivíduos envolvidos no processo realmente a percebem como necessária. Daí a necessidade de ferramentas de Gestão de Mudança que minimizem a resistência dos colaboradores, minimizem o impacto do projeto e apontem soluções aos possíveis problemas que surjam em sua implantação.

O processo de mudança organizacional, observado sob o ponto de vista da passagem de conhecimento, seja inter ou intra-organizacional, é constantemente relacionado com a cultura da organização (LAUBENGAIER et al., 2019). Cultura esta que, segundo FIGUEIREDO (2005), é um conjunto de crenças, tradições, valores, regras escritas e não escritas que podem impulsionar, acelerar, debilitar, retardar, facilitar, comprometer, dificultar ou impedir mudanças e desempenho das organizações.

Na área de serviços, por exemplo, DURYAN et al. (2018) entendem que, se o sistema adotado para gestão de conhecimento pretende facilitar sua transmissão tácita ou cultivar comunidades de prática, no processo de design do serviço, devem ser definidos os comportamentos e meios físicos para garantir a passagem de conhecimento. Esta constatação se assemelha ao já clássico problema da Engenharia de Produção: a adoção de novas tecnologias. É necessária integração efetiva entre a cultura organizacional e a estratégia corporativa em curso numa determinada organização, de forma a adequar e/ou dar suporte a inovação (SKINNER, 2006). 
Segundo o Manual de Oslo (OCDE, 1997), “uma inovação é a implementação de um produto (bem ou serviço) novo ou significativamente melhorado, ou um processo, ou um novo método de marketing, ou um novo método organizacional nas práticas de negócios, na organização do local de trabalho ou nas relações externas. ” Por definição a inovação traz consigo a mudança do estado atual das coisas. A mudança envolve transformação, interrupção e ruptura e se realiza em três etapas principais: a primeira, de desligamento das antigas práticas; a segunda, como mudança em si; e por fim, a utilização das novas práticas incorporadas à organização (CHIAVENATO, 1994). ANDERSON et al. (2001) ressaltam a importância da criação de equipes permanentes voltadas para a gestão da mudança, tendo em vista sua necessidade estratégica no cenário de constantes transformações em que as empresas estão inseridas.

O ambiente em que é aplicada a Gestão de Mudança também interfere diretamente em seu modelo de planejamento e implantação. Um projeto de gestão do conhecimento de produção artística, por exemplo, revela-se um desafio complexo devido às peculiaridades do meio: os colaboradores, muitas vezes acostumados somente a criar e produzir, podem resistir ao novo processo de colaborar e registrar conhecimento. Outro aspecto é o direito autoral que o profissional artístico quer imprimir em seus trabalhos, muitas vezes exigindo exclusividade por acreditar ser seu diferencial. Neste tipo de ambiente, a Gestão de Mudança é uma ferramenta coadjuvante que pode ser determinante para o êxito ou o insucesso do projeto.

Em projetos de edificações, as inovações podem surgir da evolução tecnológica dos softwares de representação gráfica. A modelagem digital de edificação (BIM) vai além do desenho e permite reunir compatibilização, planejamento e gestão numa mesma plataforma, modificando de forma significativa o processo de projeto. Neste contexto se faz necessário estabelecer estratégias de Gestão de Mudança para garantir eficaz implantação do BIM.

Se estas ferramentas mais recentes de gestão da mudança são de fácil acesso para os que dela precisam é a questão que se coloca, pois apenas uma parcela dos artigos publicados por pesquisadores é de open access, com acesso gratuito na web.

Este artigo tem como objetivo identificar entre as publicações acadêmicas open access as mais recentes recomendações comuns aos diferentes campos de estudo sobre Gestão de Mudança em Projetos de Inovação. Como se trata de tema amplo e multidisciplinar, opta-se por aplicar uma revisão sistemática de literatura terciária a partir das revisões de literatura publicadas sobre o assunto entre 2010 a 2019. O texto se estrutura em uma introdução ao tema, na explanação da metodologia utilizada na investigação, na exposição dos resultados obtidos, de uma breve análise destes resultados, e por fim, em uma conclusão que aponta a possibilidade de novas investigações.

A revisão sistemática de literatura (RSL), método de pesquisa utilizado neste artigo, permite desenvolver uma síntese abrangente sobre o tema e reinterpretar os resultados de pesquisas anteriores (SEURING \& GOLD, 2012). Como proposto por TORRACO (2005) essa RSL objetiva contribuir com a sistematização de um tópico de interesse no presente estudo Gestão de Mudança em Projetos de Inovação e com a entrega de uma agenda de pesquisa.

\section{METODOLOGIA}

A RSL tem sido uma ferramenta chave no desenvolvimento de base de evidências (TRANFIELD et al., 2003) e se tornou uma prática frequente para o uso dessas evidências em pesquisas. KITCHENHAM et al. (2010) consideram "que os estudos secundários podem desempenhar um papel vital tanto no apoio a mais esforços de pesquisa quanto no fornecimento de informações sobre o impacto de métodos e ferramentas para auxiliar os 
profissionais e gerentes. ” A revisão sistemática de literatura (RSL) permite a compreensão sobre o estado de arte de um determinado tema. Aqui é usada para identificar a literatura sobre a produção acadêmica relacionada à processos de Gestão de Mudança, à Projetos de Inovação e à conexão entre ambos os temas.

Para conduzir a revisão sistemática de literatura deste artigo são considerados os passos detalhados por THOMÉ et al. (2016), devidamente adaptados para a revisão terciária desenvolvida nesta pesquisa: (1) planejamento e formulação do problema; (2) busca pela literatura; (3) coleta de dados; (4) avaliação qualitativa; (5) análise dos dados e síntese; (6) interpretação; (7) apresentação de resultados; (8) atualização da revisão.

Estabelecido o escopo da pesquisa, é formulada a questão a ser considerada na busca pela literatura: Quais são as principais recomendações dos artigos de revisões de literatura open access focadas na Gestão de Mudança em Projetos de Inovação?

Estabelecido o planejamento inicial, são definidos os critérios de busca: em torno do tema "gestão" são escolhidas as palavras "management", "organizational" e "strategy", e em torno do tema "inovação" são escolhidas "innovation” e "change”. Dessa seleção resulta a seguinte estrutura de palavras-chave em operadores boleanos: "literature review" and ("innovat* manag*" or "chang* manag*" or "strateg* manag*" or "organi\#at* chang*"). A busca pela literatura é realizada em 13 de maio de 2019 nas bases Scopus e Web of Science.

Os critérios de exclusão/inclusão aplicados através de filtros são: publicações dos últimos 10 anos, textos disponíveis na íntegra e open access (acesso gratuito), revisões de literatura, no idioma português ou inglês.

São encontrados 463 artigos na base Scopus e 508 artigos na Web of Science, totalizando de 971 artigos das duas bases. Excluindo-se os artigos pagos, tem-se 175 artigos open access, sendo 73 da base Scopus e 102 da base Web of Science. Excluindo-se os repetidos nas duas bases (42), tem-se um total de 133 artigos gratuitos. Os critérios de exclusão/inclusão aplicados nos 133 artigos através da leitura do seu título e seu resumo são: artigos que tenham revisão de literatura como metodologia (sistemática ou não). A seguir, títulos e resumos são analisados por, no mínimo, dois pesquisadores; processo pelo qual se identificam 74 artigos de revisão de literatura.

Os critérios de elegibilidade definidos para análise do conteúdo são: artigos relacionados ao tema Gestão de Mudança em Projetos de Inovação. Títulos e resumos dos 74 artigos são analisados por, no mínimo, 2 pesquisadores. 51 artigos são considerados elegíveis para análise do conteúdo. A Figura 1 resume essas etapas e o quantitativo de artigos resultantes em cada uma delas.

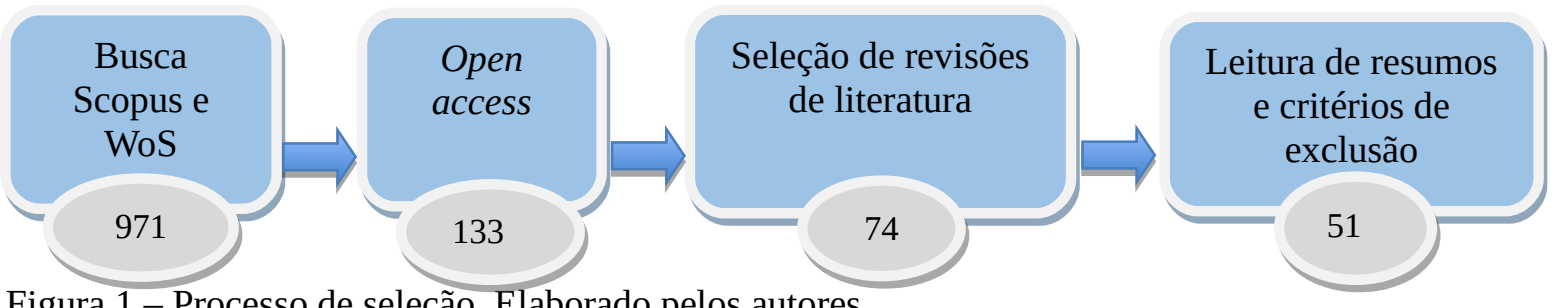

Figura 1 - Processo de seleção. Elaborado pelos autores

Nos 51 artigos selecionados são aplicadas ferramentas bibliométricas para caracterização do conjunto considerando ano de publicação, países das instituições de origem, universidades brasileiras que mais publicaram, publicação em revistas ou congressos e artigos mais citados. A interpretação dos dados coletados é apresentada no item 3.1. Para identificar as publicações mais relevantes foi utilizado o cálculo do índice interquartílico (IQR) conforme apresentado por DAWSON (2011). A partir do número de citações de cada artigo são calculados os quartis dos períodos de 2010 a 2014 e do período de 2015 a 2019 . A 
análise de conteúdo é apresentada no item 4.

\section{RESULTADOS}

\subsection{ANALISE BIBLIOMÉTRICA:}

A Figura 2 mostra a quantidade de artigos publicados por ano. Há um aumento de publicações relacionadas ao tema a partir de 2014 com aumento significativo em 2018. Em 2019, o número de publicações já equivale aos 3 primeiros anos pesquisados. Os dados de 2019 são parciais, até a data da pesquisa nas bases (13/05/2019), lembrando que são considerados apenas as publicações open access (de acesso gratuito).

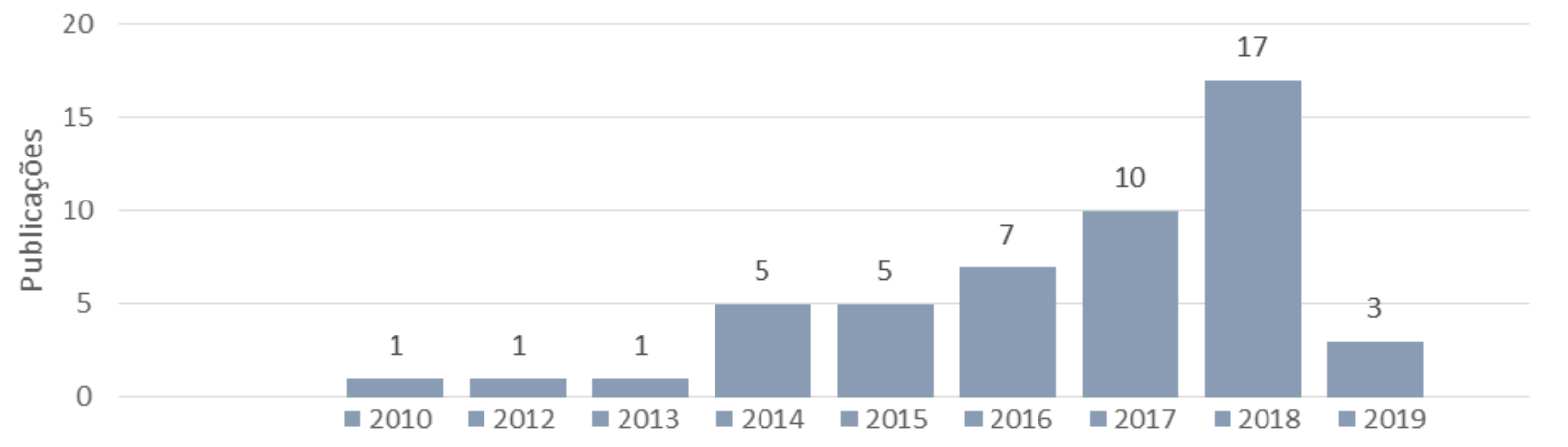

Figura 2 - Publicações por ano. Elaborado pelos autores

A Figura 3 mostra o número de artigos por país. É considerado o país da instituição de pesquisa a que pertence o autor e, no caso de autores de países diferentes, todos os países são contabilizados. Mais de $17 \%$ dos autores são de universidades brasileiras e não são encontrados artigos de autores de universidades americanas. A hipótese que justifica esta aparente distorção é que a pesquisa considera apenas publicações open access. Disto conclui-se que um número considerável de pesquisas de universidades brasileiras sobre Gestão de Mudança em Projetos de Inovação tem acesso gratuito na web. Países de excelência em pesquisa como os EUA não estão representados, sugerindo que seus artigos sobre o tema são publicados apenas em bases de acesso pago.

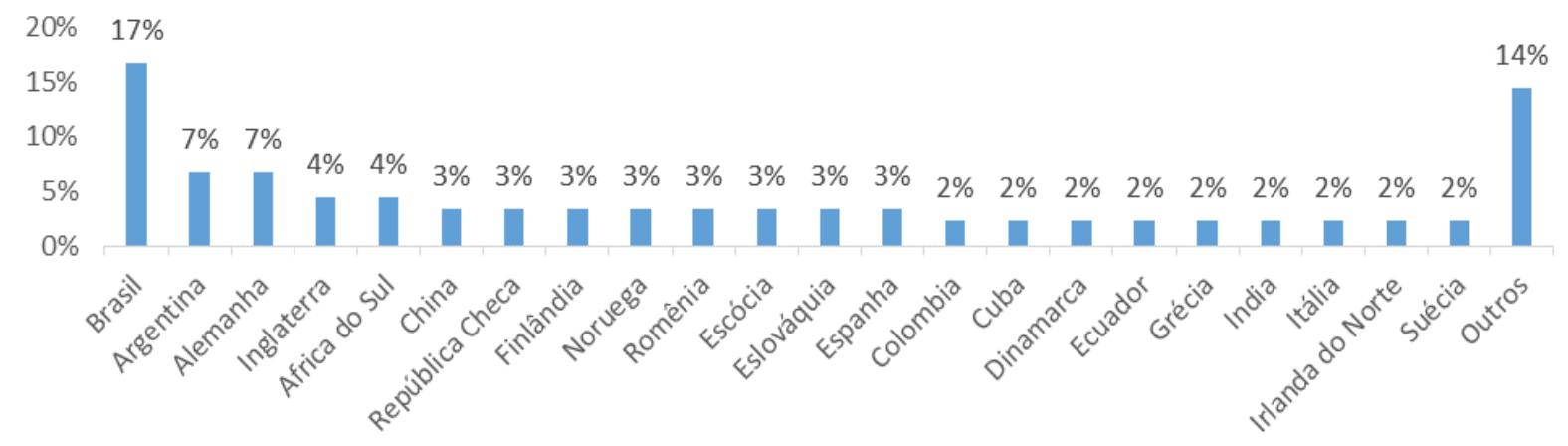

Figura 3 - Países por publicações. Elaborado pelos autores

A Figura 4 apresenta as universidades brasileiras que publicaram artigos open access sobre o tema. As universidades que mais publicam são UFMG, UNINOVE, UNISINOS e USP. A maioria dos artigos, portanto, é proveniente das regiões sul e sudeste, com maior concentração de autores provenientes de universidades do Estado de São Paulo. 


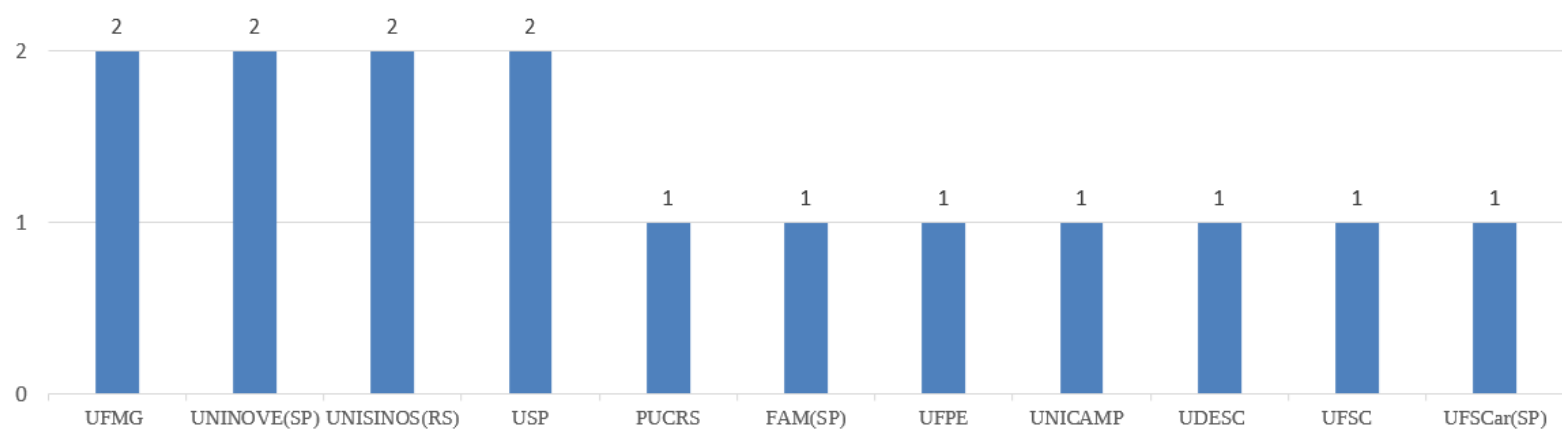

Figura 4 - Universidades brasileiras que mais publicaram. Elaborado pelos autores

Dos 51 artigos selecionados, 41 foram publicados em revistas e 9 em congressos. As revistas que mais publicaram artigos open access sobre o tema foram: Sustainability (2 artigos, em 2018 e 2019); e Amfiteatru Economic (2 artigos, em 2015 e 2017). Os congressos que mais publicaram artigos no tema, foram: 8th International Strategic Management Conference (Espanha, 2012) e 9th International Conference on Digital Enterprise Technology (China, 2016).

\begin{tabular}{|c|l|}
\hline Categoria & \multicolumn{1}{c|}{ Conclusões da presente pesquisa } \\
\hline Ano de publicação & Aumento crescente de publicações sobre o tema, especialmente em 2018. \\
\hline País dos autores & $\begin{array}{l}\text { Brasil possui maior número de autores com artigos open access sobre o } \\
\text { tema sugerindo que países de excelência em pesquisa som EUA tenham } \\
\text { suas publicações em bases pagas. }\end{array}$ \\
\hline $\begin{array}{c}\text { Universidades } \\
\text { brasileiras }\end{array}$ & $\begin{array}{l}\text { Maior concentração de artigos na região Sul/Sudeste. Maior número de artigos } \\
\text { procedentes de universidades paulistas. }\end{array}$ \\
\hline Jornal/Conferência & $\begin{array}{l}\text { Maior parte dos artigos open access sobre o tema foi publicada em revis- } \\
\text { tas. }\end{array}$ \\
\hline
\end{tabular}

Quadro 1 - Resumos da análise bibliométrica - Elaborado pelos autores.

\section{ANÁLISE DE CONTEÚDO}

\subsection{SELEÇÃO DOS ARTIGOS PARA ANÁLISE DE CONTEÚDO}

Para selecionar os artigos de maior relevância e realizar a análise de conteúdo, é considerada a quantidade de citações por artigo. Seguindo o cálculo do índice interquartílico (IQR) conforme DAWSON (2011), foram identificados os artigos considerados mais relevantes tendo como base o cálculo do quartil 1 (mínimo), quartil 2 (mediano) e quartil 3 (máximo). O índice é encontrado calculando a diferença entre o quartil mínimo e máximo. Como os artigos mais antigos apresentam mais citações, para reduzir distorções no resultado é realizada uma divisão da década para cálculo dos quartis. No período 2009 a 2014, tem-se os seguintes valores: Q1= 4,25 / Q2= 10,5 / Q3= 11,75 / IQR= 7,5 / upper limit=23. No período de 2015 a 2019, tem-se os seguintes valores: Q1=0 / Q2=0 / Q3=2 / IQR= 2 / upper limit $=5$

Este cálculo resulta em 6 artigos grifados nos Quadros 2 e 3. O Quadro 2 mostra os artigos do período de 2009 a 2014 com destaque para publicações acima do upper limit de 23 citações. Nesse sentido, apenas BOONSTRA \& BROEKHUIS (2010), com 270 citações, estão dentro da margem superior e são considerados para análise de conteúdo. O Quadro 3 mostra os artigos do período de 2015 a 2019. O cálculo do IQR resulta em upper limit de 5 citações. Cinco artigos estão dentro da margem superior e qualificados para análise de conteúdo. Observa-se que os artigos mais citados do período mais recente, 2015 a 2019, tem mais citações que o $2^{\circ}$ artigo mais citado do período de 2010 a 2014, sugerindo maior 
colaboração entre as pesquisas realizadas nos últimos cinco anos.

\begin{tabular}{|l|l|c|}
\hline \multicolumn{1}{|c|}{ Autores } & \multicolumn{1}{c|}{ Origem (periódico/congresso) } & $\mathrm{n}^{\text {o }}$ citações \\
\hline $\begin{array}{l}\text { BOONSTRA \& BROEKHUIS } \\
\text { (2010) }\end{array}$ & BMC Health Services Research & $\mathbf{2 7 0}$ \\
\hline MOLINA-AZORIN (2014) & BRQ-Business Research Quarterly & 12 \\
\hline LINDAHL et al. (2014) & $\begin{array}{l}\text { 6th CIRP Conference On Industrial Product-Service } \\
\text { Systems }\end{array}$ & 11 \\
\hline ZIZLAVSKY (2014) & Journal of Technology Management and Innovation & 11 \\
\hline DA SILVA et al. (2014) & Produção & 10 \\
\hline CLOSS \& FERREIRA (2012) & Gestão da Produção & 8 \\
\hline SIMSIT et al. (2014) & 10th International Strategic Management Conference 2014 & 3 \\
\hline DIMITRIOS et al. (2013) & 2nd International Conference On Integrated Information & 1 \\
\hline
\end{tabular}

Quadro 2 - Autores por período. Elaborado pelos autores

\begin{tabular}{|c|c|c|}
\hline Autores & $\begin{array}{c}\text { Origem } \\
\text { (periódico/congresso) }\end{array}$ & $\mathrm{n}^{\mathrm{o}}$ citações \\
\hline BAINES et al. (2017) & $\begin{array}{l}\text { International Journal Of Operations \& Production } \\
\text { Management }\end{array}$ & 59 \\
\hline SAVINO et al. (2017) & International Journal Of Management Reviews & 35 \\
\hline KNOSKOVA (2015) & Amfiteatru Economic & 9 \\
\hline DURST et al. (2015) & $\begin{array}{l}\text { Investigaciones Europeas De Direccion Y Economia De La } \\
\text { Empresa }\end{array}$ & 8 \\
\hline MORONI et al. (2015) & $\begin{array}{l}\text { 6th International Conference On Applied Human Factors } \\
\text { And Ergonomics }\end{array}$ & 7 \\
\hline MAIER et al. (2017) & 9th International Conference On Digital Enterprise Technology & 4 \\
\hline JOFFRE et al. (2017) & Aquaculture & 4 \\
\hline LÓPEZ A et al. (2016) & Informacion Tecnologica & 4 \\
\hline KUMAR et al. (2017) & International Journal Of Strategic Property Management & 4 \\
\hline MAIER et al (2016) & $\begin{array}{l}\text { International Journal Of Innovation And Sustainable } \\
\text { Development }\end{array}$ & 2 \\
\hline MOR et al. (2018) & Journal Of Operations And Supply Chain Management & 2 \\
\hline $\begin{array}{l}\text { PEREZ-PENALVER et al } \\
(2018)\end{array}$ & Journal Of Industrial Engineering And Management & 2 \\
\hline TER HAAR (2018) & Intangible Capital & 2 \\
\hline PACHECO et al. (2016) & Rege-Revista De Gestão & 1 \\
\hline KOCH et al. (2016) & $\begin{array}{l}\text { Research And Innovation In Manufacturing: Key Enabling } \\
\text { Technologies For The Factories }\end{array}$ & 1 \\
\hline EICKER et al. (2017) & Journal Of Transport And Supply Chain Management & 1 \\
\hline ADAMEK et al. (2018) & European Journal Of Sustainable Development & 1 \\
\hline ALKHORAIF et al. (2018) & Journal Of King Saud University - Engineering Sciences & 1 \\
\hline
\end{tabular}

Quadro 3 - Autores por período. Elaborado pelos autores

\subsection{PRINCIPAIS RESULTADOS DAS REVISÓES DE LITERATURA SELECIONADAS}

Os 6 artigos selecionados mostram conteúdos generalizáveis que podem ser empregados na implantação de inovações em diferentes áreas.

BOONSTRA \& BROEKHUIS (2010) descrevem as barreiras de resistência às mudanças na implementação de prontuário eletrônico de pacientes em hospitais a fim de fornecer às implementadoras opções de intervenção. As barreiras são categorizadas em: 
organizacionais; relativas ao processo de mudança; financeiras; técnicas; temporais; e psicológicas. Para quebrar os paradigmas necessários à mudança, BOONSTRA \& BROEKHUIS (2010) consideram necessário entender que não existe um caminho único para implementar inovação. Compreender o ambiente e as condições reais deve servir de base para a tomada de decisão sobre quais inovações realizar, quando e como.

BAINES et al. (2017) analisa a base de conhecimento de servitização sob a perspectiva de mudança organizacional, identificando tópicos desenvolvidos, em desenvolvimento e não desenvolvidos para fornecer uma plataforma que direcione pesquisas futuras. A análise sugere uma crescente conscientização global sobre a importância dos serviços para os fabricantes. Destacam que algumas abordagens teóricas de gerenciamento de mudança focam em parâmetros muito específicos, deixando de lado uma visão mais ampla, sistêmica e holística. Apenas algumas abordagens reúnem simultaneamente variáveis de conteúdo, contexto e processo. Segundo BAINES et al. (2017), é fundamental entender como a mudança acontece (abordagem descritiva) e como mudar (abordagem prescritiva).

SAVINO et al. (2017) destacam ferramentas internas e externas aos limites organizacionais das empresas necessárias à inovação pelo processo de busca e recombinação. A variedade e a diversidade de elementos do conhecimento são críticas na criação de inovações. Internamente à empresa, a construção de capital social e o envolvimento da equipe no processo são as formas mais efetivas de reduzir obstáculos e partilhar conhecimento entre os colaboradores. A reconfiguração corporativa é uma forma mais estruturada de promover as novas combinações de conhecimento entre colaboradores: módulos que agrupam pessoas com base nas suas competências compartilhadas; estruturas baseadas em projeto onde os colaboradores são alocados de acordo com a evolução de suas competências; e tomada de decisão centralizada. Externamente à empresa, SAVINO et al. (2017) destacam o conhecimento científico que é explorado por grandes empresas com alto investimento em pesquisa e desenvolvimento. A recombinação também pode ser explorada através de parceiros externos, seja através de alianças, aquisições, plataformas open source, consultores e coletivos. Ultrapassar barreiras geográficas também pode aumentar as fontes e variedade de conhecimento para reconfiguração e inovação.

Segundo KNOSKOVA (2015), inovadores radicais usam sistemas de inovação eficientes no desenvolvimento de produtos, incluindo avaliação de tendências tecnológicas e tendências de mercado. A principal abordagem de KNOSKOVA (2015) é o empreendedorismo corporativo. Destacam-se os conceitos do empreendedorismo, intraempreendedorismo e inovação que são mutuamente inclusivos. O empreendedor na era do conhecimento deve buscar a inovação como diferencial competitivo através de mudanças constantes. Inovação é questão complexa e envolve gerenciamento de vários fatores diferentes. Empresas tradicionais tem mais dificuldade de sobreviver a inovações radicais do que novas empresas em virtude dos seus compromissos pré-existentes. Segundo KNOSKOVA (2015) a cultura empreendedora incentiva inovadores radicais e propicia o desenvolvimento de novos produtos, especialmente na flexibilidade de pequenos projetos que através de rápida experimentação e feedbacks são capazes de orientar para novas direções.

Em sua revisão bibliográfica, DURST et al. (2015) ressaltam o efeito positivo da inovação na eficácia operacional e na eficiência de custos, resultando em maior produtividade e aumento da qualidade dos serviços. Entretanto, DURST et al. (2015) destacam resultados em que mostram que firmas focadas em inovação de serviços têm maior produtividade (vendas por empregados) mas têm seus ganhos neutralizados por aumentos nos custos. Segundo DURST et al. (2015), mecanismos de governança podem ser utilizados para fomentar inovação de forma horizontal, promovendo a coordenação entre parceiros e mitigando o oportunismo. 
MORONI et al. (2015), o artigo proveniente de universidade brasileira, destaca 3 passos para o design orientado por inovação em startups: ouvir, interpretar e divulgar. Ouvir se refere a entender o potencial de sucesso, identificar seus intérpretes, integrar seus intérpretes e organizar encontros para reuni-los. Interpretar se refere a reunir informações e registrar ideias, integrar ideias externas com as internas à empresa, analisar as necessidades dos usuários, desenvolver novos conceitos e significados, desenvolver estratégia de inovação. Divulgar por sua vez se refere a elaborar um protótipo, desenvolver o produto e elaborar plano de comunicação para os novos conceitos que incluam a divulgação do produto/serviço através da rede de intérpretes.

O Quadro 4 apresenta o resumo dos 6 artigos considerados mais relevantes devido a um número de citações acima do upper limit na ordenação dos artigos em quartis.

\begin{tabular}{|c|c|}
\hline Autor & Contribuição \\
\hline $\begin{array}{c}\text { BOONSTRA \& } \\
\text { BROEKHUIS (2010) }\end{array}$ & $\begin{array}{l}\text { Categorização das barreiras à inovação; ambiente e condições reais } \\
\text { devem embasar decisão sobre quando e como inovar. }\end{array}$ \\
\hline BAINES et al. (2017) & $\begin{array}{l}\text { É fundamental entender como a mudança acontece (abordagem descri- } \\
\text { tiva) e como mudar (abordagem prescritiva). }\end{array}$ \\
\hline SAVINO et al. (2017) & $\begin{array}{l}\text { Variedade e a diversidade de conhecimento são críticos para inovar; } \\
\text { processo de busca e recombinação como forma de gerar inovação. }\end{array}$ \\
\hline KNOSKOVA (2015) & $\begin{array}{l}\text { Identificação de fatores organizacionais e de processos que apoiam } \\
\text { inovações radicais. }\end{array}$ \\
\hline DURST et al. (2015) & Inovação possibilita aumentar produtividade e qualidade dos serviços. \\
\hline MORONI et al. (2015) & Identificação dos 3 passos para criar conceitos inovadores de negócio. \\
\hline
\end{tabular}

\section{CONCLUSÃO}

A pesquisa encontra uma quantidade significativa de revisões bibliográficas sobre o tema Gestão de Mudança em projetos de inovação. A seleção final de artigos revela uma série de resultados tanto descritivos, observados na implantação de inovações pesquisadas, como prescritivos, na forma de recomendações para implantação de inovação. Estes resultados além acessíveis na web sem custo, revelam-se consideravelmente generalizáveis aos diferentes campos de inovação.

Entre as principais recomendações para a Gestão de Mudança em Projetos de Inovação estão: a compreensão do ambiente e das condições reais como ponto de partida para a inovação (BOONSTRA et al., 2010); a necessidade de entender como a mudança acontece e como mudar (BAINES et al., 2017); o efeito positivo da inovação na eficácia operacional e na eficiência de custos (DURST et al. (2015), a importância da construção do capital social interno e de alianças e parcerias externas (SAVINO et al., 2017); a cultura empreendedora como fonte de inovação (KNOSKOVA, 2015); e os passos para inovação estabelecidos por MORONI et al. (2015).

As principais lacunas de pesquisa identificadas são: desenvolver abordagens holísticas de mudança que reúnam simultaneamente variáveis de conteúdo, contexto e processo. (BAINES et al.,2017); realizar novos estudos de caso para explorar o processo de busca e recombinação em pequenas e medias empresas (SAVINO et al., 2017), investigar sobre as capacidades necessárias as empresas tradicionais para sobrevivência às inovações radicais (KNOSKOVA, 2015), pesquisar sobre a possibilidade da inovação gerar maior produtividade, mas neutralizar os ganhos por aumentos nos custos. (DURST et al., 2015), e estudar alternativas para que pequenas empresas posam entender o ambiente externo da sua organização para encontrar parcerias que possam contribuir para a geração de inovações. (MORONI et al., 2015). 
Sugerimos também como itens de pesquisa futura a atualização da revisão bibliográfica proposta por THOMÉ et al. (2016), a análise de conteúdo das 51 RSL encontradas sobre o tema e a proposição de uma estrutura conceitual como sugerido por TORRACO (2005).

\section{REFERÊNCIAS BIBLIOGRÁFICAS}

[1] ADAMEK, Pavel. An investigation of interconnection between business excellence models and corporate sustainability approach. European Journal of Sustainable Development, Rome: European Center of Sustainable Development, v. 7, n. 1, p. 381394, 2018.

[2] ALISHIRI, Mohammad Javad; MAKVANDI, Payam; KHAMESH, Abbas. Identification and Ranking the Critical Success Factors of Business Incubator of Science and Technology Parks-A Case Study: Business Incubator of Baqiyatallah University of Medical Sciences. Journal of Applied Biotechnology Reports, [Teerã]: [Baqiyatallah University of Medical Sciences], v. 5, n. 2, p. 64-69, 2018.

[3] ALKHORAIF, Abdullah; MCLAUGHLIN, Patrick. Lean implementation within manufacturing SMEs in Saudi Arabia: Organizational culture aspects. Journal of King Saud University-Engineering Sciences, [Amsterdã]: Elsevier, v. 30, n. 3, p. 232242, 2018.

[4] ANDERSON, D.; ANDERSON, L. S. Beyond Change Management: Advanced Strategic for Today's Transformational Leaders. San Francisco: Jossey-Bass, 2001.

[5] BAINES, Tim et al. Servitization: revisiting the state-of-the-art and research priorities. International Journal of Operations \& Production Management, [Reino Unido]: Emerald Publishing, v. 37, n. 2, p. 256-278, 2017.

[6] BOONSTRA, Albert; BROEKHUIS, Manda. Barriers to the acceptance of electronic medical records by physicians from systematic review to taxonomy and interventions. BMC health services research, [London]: [BioMed Central], v. 10, n. 1, p. 231, 2010.

[7] CHEPA, Noraziah; JASIN, Noorhayati Md; BAKAR, Nur Azzah Abu. How change management can prevent the failure of information systems implementation among Malaysian government hospitals? In: AIP Conference Proceedings. [Estados Unidos]: AIP Publishing, 2017. p. 020037.

[8] CHIAVenato, I. Gestão De Pessoas: O Novo Papel dos Recursos Humanos nas Organizações. Rio de Janeiro: Elsevier, 1999.

[9] CLOSS, Lisiane Quadrado; FERREIRA, Gabriela Cardozo. University-industry technology transfer in the Brazilian context: a review of scientific studies published from 2005 to 2009. Gestão \& Produção, [São Paulo]: [Universidade Federal de São Carlos Departamento de Engenharia de Produção], v. 19, n. 2, p. 419432, 2012.

[10] CURVELlO, J. J. A. Comunicação Interna e Cultura Organizacional. São Paulo: Scortecci, 2002.

[11] DAWSON, R. How significant is a boxplot outlier? Journal of Statistics Education v. 19, n. 2, p. 1-13, 2011. 
[12] DIMITRIOS, Nasiopoulos K.; SAKAS, Damianos P.; VLACHOS, D. S. Analysis of strategic leadership models in information technology. Procedia-Social and Behavioral Sciences, [Oxford]: Elsevier, v. 73, p. 268-275, 2013.

[13] DURST, Susanne; MENTION, Anne-Laure; POUTANEN, Petro. Service innovation and its impact: What do we know about? Investigaciones europeas de dirección y economía de la empresa, [Espanha]: Elsevier España, v. 21, n. 2, p. 65-72, 2015.

[14] DURYAN, Meri; SMYTH, Hedley. Service design and knowledge management in the construction supply chain for an infrastructure programme. Built Environment Project and Asset Management, [Reino Unido]: Emerald Publishing, v. 9, n. 1, p. 118-137, 2019.

[15] EICKER, Themari; CILLIERS, Jacoba O. Equipping small business retailers to manage logistical supply chain drivers: A theoretical guideline. Journal of Transport and Supply Chain Management, [Cidade do Cabo]: AOSIS, v. 11, n. 1, p. 112, 2017.

[16] FIGUEIREDO, Saulo Porfírio. Gestão do Conhecimento: Estratégias competitivas para a criação e mobilização do conhecimento na empresa: descubra como alavancar e multiplicar o capital intelectual e o conhecimento da organização. Rio de Janeiro: Ed. Qualitymark, 2005.

[17] GARCEZ, Marcos Paixao; MACCARI, Emerson Antonio. Evaluation Methodology for The R\&D Project Portfolio By Using The Risk Adjusted Net Present ValueOne Case Study In The Petrochemical Industry. Revista de Gestao e Projetos, [São Paulo]: [Universidade Nove de Julho], v. 6, n. 2, p. 1, 2015.

[18] HAAR, Philipp ter. Measuring innovation: A state of the science review of existing approaches. Intangible Capital, [Barcelona]: [Intangiblecapital.org], v. 14, n. 3, p. 409-428, 2018.

[19] JOFFRE, Olivier M. et al. How is innovation in aquaculture conceptualized and managed? A systematic literature review and reflection framework to inform analysis and action. Aquaculture, [Países Baixos]: Elsevier, v. 470, p. 129-148, 2017.

[20] KITCHENHAM, Barbara et al. Systematic literature reviews in software engineering - a tertiary study. Information and software technology, [Países Baixos]: Elsevier, v. 52, n. 8, p. 792-805, 2010.

[21] KNOŠKOVÁ, L’ubica. Innovation processes and entrepreneurial culture for radical innovations. Amfiteatru Economic Journal, [Bucareste]: [Editura ASE], v. 17, n. 38, p. 342-357, 2015.

[22] KOCH, Jonas; MICHELS, Nicolas; REINHART, Gunther. Context model design for a process-oriented manufacturing change management. Procedia CIRP, [Amsterdã]: Elsevier, v. 41, p. 33-38, 2016.

[23] KRÜGER, Louis LSJ; PRETORIUS, Jan Harm C.; ERASMUS, Louwrence D. Towards a Comprehensive Systematic Innovation Model: A Literature review. SAIEE Africa Research Journal, [Africa do Sul]: South African Intitute of Electrical Engineers, v. 110, n. 1, p. 39-46, 2019.

[24] KUMAR, Anil et al. Evaluating innovation capabilities of real estate firms: a combined fuzzy Delphi and DEMATEL approach. International Journal of Strategic 
Property Management, [Vilnius]: Vilnius Gediminas Technical University Press, v. 21, n. 4, p. 401-416, 2017.

[25] LAUBENGAIER, Désirée; HAHN, Gerd; WAGNER, Heinz-Theo. Organizational Culture and Knowledge Exchange and Combination: A Systematic Literature Review. In: Proceedings of the 52nd Hawaii International Conference on System Sciences, [Honolulu]: [University of Hawaii at Manoa], p. 5498-5507, 2019.

[26] LINDAHL, Mattias; SAKAO, Tomohiko; CARLSSON, Emma. Actor's and System Maps for Integrated Product Service Offerings-Practical Experience from Two Companies. Procedia CIRP, [Amsterdã]: Elsevier, v. 16, p. 320-325, 2014.

[27] LÓPEZ, Ana et al. Desarrollo e Instrumentación de un Proceso de Vigilancia Tecnológica basado en Protocolos de Revisión Sistemática de la Literatura. Información tecnológica, [La Serena]: [Centro de Información Tecnológica], v. 27, n. 4, p. 155-164, 2016.

[28] LV, Wen-Dong et al. Innovation Resilience: A New Approach for Managing Uncertainties Concerned with Sustainable Innovation. Sustainability, [Basileia]: MDPI, v. 10, n. 10, p. 3641, 2018.

[29] MAIER, Dorin et al. Development and operationalization of a model of innovation management system as part of an integrated quality-environment-safety system. Amfiteatru Economic Journal, [Bucareste]: [Editura ASE], v. 19, n. 44, p. 302-314, 2017.

[30] MAIER, Maximilian A.; BREM, Alexander; KAUKE, Matthias. Exploring the boundaries of corporate social responsibility and innovation: a conceptual framework of socio-political stakeholders and their integration into the innovation process. International Journal of Innovation and Sustainable Development, v. 10, n. 3, p. 312-337, 2016.

[31] MAYAKUL, T.; KIATTISIN, S.; PRASAD, R. A Sustainable Medical Tourism Framework Based on the Enterprise Architecture Design: The Case in Thailand. Journal of Green Engineering, [India]: River Publishers, v. 8, n. 3, p. 359388, 2018.

[32] MENDES, Marco Aurélio de Souza; BAX, Marcello Peixoto. BPM and ECM: Similarities, differences, conceptual, and technological limits. Transinformação, Campinas: [Pontifícia Universidade Católica de Campinas Centro de Ciências Humanas e Sociais Aplicadas Faculdade de Biblioteconomia], v. 30, n. 1, p. 95-105, 2018.

[33] MENG, Xianhai; BROWN, Andrew. Innovation in construction firms of different sizes: drivers and strategies. Engineering, Construction and Architectural Management, [Reino Unido]: Emerald Publishing, v. 25, n. 9, p. 1210-1225, 2018.

[34] MINATOGAWA, Vinicius Luiz Ferraz et al. Business model innovation influencing factors: an integrative literature review. Brazilian Journal of Operations \& Production Management, [Rio de Janeiro]: Associação Brasileira de Engenharia de Produção, v. 15, n. 4, p. 610-617, 2018.

[35] MOLINA-AZORÍN, José F. Microfoundations of strategic management: Toward micro-macro research in the resource-based theory. BRQ Business Research Quarterly, [Barcelona]: Elsevier España, v. 17, n. 2, p. 102-114, 2014. 
[36] MOR, Rahul S.; BHARDWAJ, Arvind; SINGH, Sarbjit. A structured-literaturereview of the supply chain practices in dairy industry. Journal of Operations and Supply Chain Management, São Paulo: FGV EAESP, v. 11, n. 1, p. 14-25, 2018.

[37] MORONI, Isabela; ARRUDA, Amilton; ARAUJO, Kátia. The design and technological innovation: how to understand the growth of startups companies in competitive business environment. Procedia Manufacturing, [Amsterdã]: Elsevier, v. 3, p. 2199-2204, 2015.

[38] NEL, Charles Benjamin Hirschowitz; JOOSTE, J. L. A technologically-driven asset management approach to managing physical assets-a literature review and research agenda for smart asset management. South African Journal of Industrial Engineering, [Africa do Sul]: [Stellenbosch University Library and Information Service], v. 27, n. 4, p. 50-65, 2016.

[39] OCDE - Organização Para Cooperação Econômica e Desenvolvimento. Manual de Oslo: Proposta de Diretrizes Para Coleta e Interpretação de Dados Sobre Inovação Tecnológica. Tradução: Paulo Garchet. [Rio de Janeiro]: FINEP, 2004.

[40] PACHECO, Fabiana B.; KLEIN, Amarolinda Z.; DA ROSA RIGHI, Rodrigo. Business models for products and services based on the internet of things: a literature review and future research opportunity. Revista de Gestão. USP, São Paulo: Universidade de São Paulo, Faculdade de Economia Administração e Contabilidade. Departamento de Administração, v. 23, n. 1, p. 41-52, 2016.

[41] PEÑALVER, María Jose Pérez; MAS, Lourdes E. Aznar; FLETA, Begoña Montero. Identification and classification of behavioural indicators to assess innovation competence. Journal of Industrial Engineering and Management, [Barcelona]: [Universitat Politècnica de Catalunya], v. 11, n. 1, p. 87-115, 2018.

[42] PETZOLD, Neele; LANDINEZ, Lina; BAAKEN, Thomas. Disruptive innovation from a process view: A systematic literature review. Creativity and Innovation Management, [Reino Unido]: John Wiley \& Sons, v. 28, n. 2, p. 157-174, 2019.

[43] PODMETINA, Daria et al. Developing a competency model for open innovation: From the individual to the organisational level. Management Decision, [Reino Unido]: Emerald Publishing, v. 56, n. 6, p. 1306-1335, 2018.

[44] PONCE, Diana Katiuska Peña; LORENZO, Angie Fernández; CONCEPCIÓN, Raúl Ricardo Fernández. Innovation Management: The Need of a Model for Manufacturing Micro-Enterprises. Quality Innovation Prosperity, [Košice]: [Technical University of Košice], v. 21, n. 3, p. 01-14, 2017.

[45] PROSTEAN, G.; VOLKER, S.; HUTANU, A. Change management methodologies trained for automotive infotainment projects. In: IOP Conference Series: Materials Science and Engineering. [Reino Unido]: IOP Publishing, v.163, p. 012012, 2017.

[46] RIVERA SILVA, Andres Camilo; VARGAS REYES, Rafael Esteban; BOHÓRQUEZ AREVALO, Luz Esperanza. Implementation of Enterprise Resource Planning (ERP) Systems in Organizations Since Coevolution. Revista Ingeniería Solidaria, [Bogotá]: Universidad Cooperativa de Colombia, v. 14, n. 24, p. 1-15, 2018.

[47] SAVASTANO, Marco et al. Contextual Impacts on Industrial Processes Brought by the Digital Transformation of Manufacturing: A Systematic Review. Sustainability, [Basileia]: MDPI, v. 11, n. 3, p. 891, 2019. 
[48] SAVINO, Tommaso; MESSENI PETRUZZELLI, Antonio; ALBINO, Vito. Search and recombination process to innovate: a review of the empirical evidence and a research agenda. International Journal of Management Reviews, v. 19, n. 1, p. 54-75, 2017.

[49] SENGE, Peter M.; GARDINI, Carlos. La quinta disciplina. Sperling \& Kupfer, 1992.

[50] SEURING, S.; GOLD, S. Conducting content-analysis based literature reviews in supply chain management. Supply Chain Management, v. 7, n. 5, p. 544-555, 2012.

[51] SILVA, Débora Oliveira da; BAGNO, Raoni Barros; SALERNO, Mario Sergio. Models for innovation management: review and analysis of the literature. Production, [São Paulo]: [Associação Brasileira de Engenharia de Produção], v. 24, n. 2, p. 477-490, 2014.

[52] ŞIMŞIT, Zeynep Tuğçe; VAYVAY, Özalp; ÖZTÜRK, Özgen. An outline of innovation management process: building a framework for managers to implement innovation. Procedia-Social and Behavioral Sciences, [Oxford]: Elsevier, v. 150, p. 690-699, 2014.

[53] SINGH, Harpreet; AMINI, Ardavan; HERNANDEZ-MUNOZ, Luis. An Enterprise Systems Model to Deliver Innovation in the Healthcare Industry Based on Cognitive and Social-Tech Engineering. Procedia-Social and Behavioral Sciences, [Oxford]: Elsevier, v. 195, p. 1705-1714, 2015.

[54] SKINNER, Wickham. Manufacturing strategy: the story of its evolution. Journal of Operations Management, [Amsterdã]: Elsevier, v. 25, n. 2, p. 328-335, 2007.

[55] SUJOVÁ, Andrea; REMEŇ, Ondrej. Management of changes in business processes: an empirical study in Slovak enterprises. Engineering Management in Production and Services, v. 10, n. 3, p. 37-50, 2018.

[56] TEZA, Pierry et al. Front end of innovation models: similarities, differences and research perspectives. Production, [São Paulo]: [Associação Brasileira de Engenharia de Produção], v. 25, n. 4, p. 851-863, 2015.

[57] THOMÉ, Antônio Márcio Tavares; SCAVARDA, Luiz Felipe; SCAVARDA, Annibal José. Conducting systematic literature review in operations management. Production Planning \& Control, [Reino Unido]: Taylor \& Francis, v. 27, n. 5, p. 408-420, 2016.

[58] TORRACO, R. J. Writing integrative literature reviews: guidelines and examples. Human Resource Development Review. v. 4, n. 3, p. 356-367, 2005.

[59] TRANFIELD, David; DENYER, David; SMART, Palminder. Towards a methodology for developing evidence-informed management knowledge by means of systematic review. British journal of management, [Reino Unido]: British Academy of Management, v. 14, n. 3, p. 207-222, 2003.

[60] UBEDA, Cristina Lourenço; SANTOS, Fernando César Almada; NAGANO, Marcelo Seido. Analysis of the individual competences contributions to innovation management based on methodological triangulation. Gestão \& Produção, [São Paulo]: [Universidade Federal de São Carlos Departamento de Engenharia de Produção], v. 24, n. 3, p. 595-609, 2017. 
[61] VELINOV, Emil; VASSILEV, Vasko; DENISOV, Igor. Holacracy and obliquity: contingency management approaches in organizing companies. Problems and Perspectives in Management. Sumy: Business Perspectives, v. 16, n. 1, p. 330-335, 2018.

[62] VILTARD, Leandro Adolfo. Corporate University: An implementation case analysis, in Argentina. Independent Journal of Management \& Production, [Bauru]: [s.n.], v. 9, n. 4, p. 1235-1253, 2018.

[63] WARDHANI, Arie Restu; ACUR, N.; MENDIBIL, Kepa. Human capital, social capital and innovation outcome: A systematic review and research agenda. In: 2016 IEEE International Conference on Industrial Engineering and Engineering Management (IEEM). [Estados Unidos]: IEEE, 2016. p. 355-359.

[64] WEERAKOON, Ranjan Kumara. Human resource management in sports: A critical review of its importance and pertaining issues. Physical Culture and Sport. Studies and Research, [Varsóvia]: [De Gruyter Poland], v. 69, n. 1, p. 15-21, 2016.

[65] ZAPATA ROTUNDO, Gerardo J.; HERNÁNDEZ ARIAS, Aymara. Absorptive capacity: a literature review and a model of its determinants. Revista de Ciencias de la Administración y Economía, [Quito]: Universidad Politécnica Salesiana del Ecuador, v. 8, n. 16, p. 121-140, 2018.

[66] ZIZLAVSKY, Ondrej. The balanced scorecard: Innovative performance measurement and management control system. Journal of technology management \& innovation, [Santiago]: Facultad de Economía Universidad Alberto Hurtado, v. 9, n. 3, p. 210-222, 2014.

As Referências Bibliográficas devem seguir as normas da ABNT/NBR 6023. 